\title{
GAIA CONFIRMS THAT SDSS J102915+172927 IS A DWARF STAR
}

\author{
P. Bonifacio, ${ }^{1}$ E. Caffau,${ }^{1}$ M. Spite,${ }^{1}$ F. Spite,${ }^{1}$ P. François, ${ }^{1}$ S. Zaggia,${ }^{2}$ F. Arenou, ${ }^{1}$ R. Haigron, ${ }^{1}$ N. Leclerc,${ }^{1}$ O. Marchal, ${ }^{1}$ \\ P. Panuzzo, ${ }^{1}$ G. Plum,${ }^{1}$ and P. Sartoretti ${ }^{1}$
}

${ }^{1}$ GEPI, Observatoire de Paris, Université PSL, CNRS, Place Jules Janssen, F-92195 Meudon, France

${ }^{2}$ Istituto Nazionale di Astrofisica, Osservatorio Astronomico di Padova, Vicolo dell'Osservatorio 5, 35122 Padova, Italy

Keywords: Stars: Population II - Stars: abundances - Galaxy: abundances - Galaxy: formation Galaxy: halo 


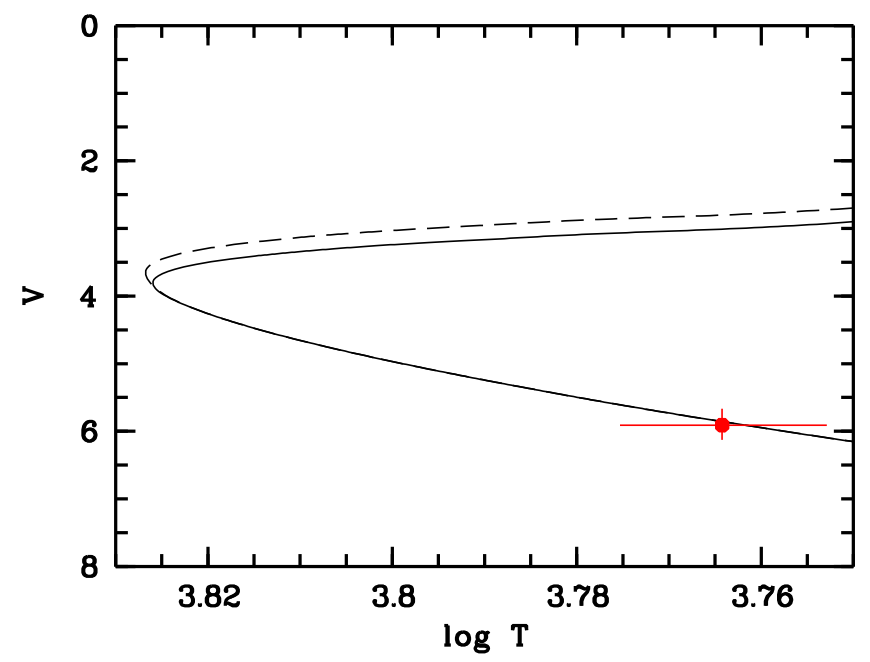

Figure 1. The absolute magnitude of SDSS J102915+172927 derived from the Gaia parallax and the Gaia photometry implies it is a dwarf star. For comparison two isochrones (A. Chieffi, private communication) computed with the FRANEC code for 14 Gyrs and $Z=2 \times 10^{-6}$ (dashed line), $Z=2 \times 10^{-5}$ (solid line) and $Z=2 \times 10^{-4}$ (dotted line) are shown.

With a global metallicity of $Z \leq 6.9 \times 10^{-7}$ (Caffau et al. 2011, 2012) star SDSS J102915+172927 is, at the time of writing, the most metal-poor object known. This star is also remarkable because it has abundances of $\mathrm{C}$ and $\mathrm{O}$ that are low enough that the metal line cooling proposed by Bromm \& Loeb (2003) cannot be efficient to allow a low-mass star to form. The very existence of this star has been taken as proof that other mechanisms, such as dust cooling (Schneider, et al. 2012) and fragmentation (Klessen, et al. 2012) can allow to form low-mass stars at metallicities below what is allowed by metal line cooling alone.

Another of the distinctive features of this star is that lithium has not been detected. This is at variance with what is observed in warm metal-poor stars of higher metallicity, which display a constant lithium abundance, the Spite plateau (Spite \& Spite 1982). As discussed in Bonifacio et al. (2018) amongst all the known unevolved stars with $[\mathrm{Fe} / \mathrm{H}]<-4.1$ only two have a measured lithium abundance and in both cases it is more than 0.2 dex below the Spite plateau. The effective temperature of SDSS J102915+172927 is $5811 \mathrm{~K}$ (Caffau et al. 2012) and for such stars stellar evolution models do not predict extensive Li depletion, as is the case for cooler stars (see e.g. Salaris \& Weiss 2001). Bonifacio et al. (2018) suggested that the effective temperature at which convection begins to effectively deplete lithium increases with decreasing $[\mathrm{Fe} / \mathrm{H}]$.

MacDonald, et al. (2013) have argued that the upper limit on the lithium abundance in SDSS J102915+172927 is stringent enough that it rules out an identification as a dwarf star. For this reason they argued that it is indeed a subgiant star. In the scenario in which the star is a dwarf, they estimate a distance of $1.35 \pm 0.16 \mathrm{kpc}$, while in the subgiant scenario they estimate a distance of $6.2 \pm 0.5 \mathrm{kpc}$. The subgiant scenario has an important corollary: it allows for the star to be formed in a C-enriched medium, where metal line cooling is effective, that has been subsequently enriched by a supernova of type Ia, that produced the low $\mathrm{C} / \mathrm{Fe}$ ratio observed.

It is very difficult to strongly constrain the luminosity of a warm low metallicity star from its spectrum. In the case of SDSS J102915+172927, for example, we do not observe any FeII line, that could constrain the luminosity, by imposing the ionization equilibrium. The broad photometric comparison with theoretical isochrones of Caffau et al. (2012) gave the dwarf as best solution with the subgiant scenario slightly less favourable. The Gaia mission (Gaia Collaboration et al. 2016) with its second data release (Gaia Collaboration et al. 2018; Arenou et al. 2018) has provided us a very accurate parallax of $0.734 \pm 0.073$ mas. Thus the distance is $1.37_{-0.12}^{+0.15} \mathrm{kpc}$, in perfect agreeement with what estimated by Caffau et al. $(2012,1.37 \pm 0.20 \mathrm{kpc})$. Gaia also provides photometry for this star $G=16.548$ and a colour $B P-R P=0.799$. The star is only slightly reddened, the reddening maps of Schlafly et al. (2017) imply $E(B-V)=0.03$ mag. We used the above information to compute the absolute $\mathrm{V}$ magnitude using the transformations provided by the Gaia DR2 documentation (https://gea.esac.esa.int/archive/documentation/GDR2/Data_processing/ chap_cu5pho/sec_cu5pho_calibr/ssec_cu5pho_PhotTransf.html). This allows us to compare it to the metal-poor 
isochrones computed by A. Chieffi (private communication) and shown in the figure. We can unambiguously conclude that SDSS J102915+172927 is a dwarf. The subgiant scenario proposed by MacDonald, et al. (2013) can be definitely ruled out, together with the hypothesis that SDSS J102915+172927 was formed from a medium dominantly enriched by SNIa. This reinforces the need for dust cooling and fragmentation to form a star like this.

This work has made use of data from the European Space Agency (ESA) mission Gaia (https://www. cosmos. esa. int/gaia), processed by the Gaia Data Processing and Analysis Consortium (DPAC, https://www . cosmos. esa.int/ web/gaia/dpac/consortium). Funding for the DPAC has been provided by national institutions, in particular the institutions participating in the Gaia Multilateral Agreement.

\section{REFERENCES}

Arenou, F., Luri, Z. et al. , 2018, A\&A, in press

arXiv: 1804.09375

Bonifacio, P., Caffau, E., Spite, M., et al. 2018, A\&A in press, arXiv:1801.03935

Bromm, V., \& Loeb, A. 2003, Nature, 425, 812

Caffau, E., Bonifacio, P., François, P., et al. 2012, A\&A, 542, A51

Caffau, E., Bonifacio, P., François, P., et al. 2011, Nature, 477,67

Gaia Collaboration, Prusti, T., de Bruijne, J. H. J., et al. 2016, A\&A, 595, A1
Gaia Collaboration, Brown, A.G.A., Vallenari A., et al. 2018, A\&A, in press, arXiv:1804.09365

Klessen, R. S., Glover, S. C. O. \& Clark, P. C. 2012, MNRAS, 421, 3217.

MacDonald, J., Lawlor, T. M., Anilmis, N., et al. 2013, MNRAS, 431, 1425.

Salaris, M., \& Weiss, A. 2001, A\&A, 376, 955

Schlafly, E. F., Peek, J. E. G., Finkbeiner, D. P., \& Green, G. M. 2017, ApJ, 838, 36

Schneider, R., Omukai, K., Limongi, M., et al. 2012, MNRAS, 423, L60.

Spite, M., \& Spite, F. 1982, Nature, 297, 483 